\title{
Off-label Non-vitamin K Antagonist Oral Anticoagulants Does Not Decrease the Stroke Risk of Atrial Fibrillation Patients in East China
}

\author{
Peng Zhou \\ Huashan Hospital Fudan University \\ Rongchen Liu \\ Huashan Hospital Fudan University \\ Yangjie Yu \\ Huashan Hospital Fudan University \\ Wei Chen \\ Huashan Hospital Fudan University

\section{Yunzhi Ma} \\ Huashan Hospital Fudan University \\ Hengyang Wang \\ Huashan Hospital Fudan University \\ Maieryemu Waresi \\ Huashan Hospital Fudan University

\section{Meng Wang} \\ Huashan Hospital Fudan University

\section{Xinping Luo} \\ Huashan Hospital Fudan University \\ Jian Li (D13816066763@163.com ) \\ Huashan Hospital Fudan University
}

\section{Research}

Keywords: Atrial fibrillation, anti-coagulation therapy, off-label dose of NOACs, cardiovascular mortality

Posted Date: January 11th, 2021

DOI: https://doi.org/10.21203/rs.3.rs-141450/v1

License: (c) (1) This work is licensed under a Creative Commons Attribution 4.0 International License.

Read Full License 


\section{Abstract}

Background: For anticoagulation therapy of atrial fibrillation (AF) in east Asia, some off-label dose, so called "Asian Dose" of non-vitamin K antagonist oral anticoagulants (NOACs) was used concerning on the bleeding risks in these patients, such as low-dose rivaroxaban (10-15 mg once daily) or dabigatran (110-150 mg once daily). However, the efficacy of the off-label dose of NOACs remains controversial.

Methods: We conducted a retrospective cohort study to compared the efficacy and safety among patients with AF in three groups: patients with the off-label dose of NOACs treatment (OFL group, dabigatran 110 $\mathrm{mg}$ once daily or rivaroxaban under $20 \mathrm{mg}$ daily), patients with the standard dose of anti-coagulation therapy (SAG group, dabigatran $110 \mathrm{mg}$ twice daily or rivaroxaban $20 \mathrm{mg}$ once daily, or warfarin with international normalized ratio (INR) 2-3), and patients without non-coagulation treatment (NCG group) in east China.

Results: A total of 296 patients were recruited in our study. Compared to patients in SAG group, patients in OFL group had higher risk in stroke and thromboembolism events $(P=0.000)$. The risk of other events including major bleeding $(P=0.597)$ was comparable in these two groups, while there was no significant difference in all-cause mortality, which were both dramatically lower than NAG group $(0.52, P=0.000)$.

Conclusions: Collectively, our results demonstrate that "Asian dose" of NOACs indeed can not bring much benefit for AF patients in east China.

\section{Introduction}

Atrial fibrillation (AF) is the most common sustained arrhythmia worldwide[1, 2], while anti-coagulation therapy remains the main strategy for $\mathrm{AF}$ including vitamin $\mathrm{K}$ antagonists (VKAs) and non-vitamin $\mathrm{K}$ antagonist oral anticoagulants (NOACs)[3]. Some studies have reported that the efficacy of NOACs are superior than VKAs among AF patients in East Asia[4].

Because of a greater risk for anti-coagulation related intracranial hemorrhage in east Asia, there is a compromised dosage of NOACs concerning the bleeding risk for anti-coagulation therapy[5]. Patients are usually given a so called "Asian dose" of NOACs such as low-dose rivaroxaban (10-15 mg once daily) or dabigatran (110-150 mg once daily) in China[6, 7]. Several studies have demonstrated that Asian dose brings much benefit for $\mathrm{AF}$ patients in East Asian due to the lower bleeding risk[4, 8]. However, the latest European Society of Cardiology (ESC) guideline indicates that reduced dose may be only favorable for patients with renal disease for rivaroxaban and apixaban[9]. The benefit of "Asian dose" was still controversial. Here, we set out this observational study to investigate whether the "Asian dose" of NOACs really exists for stroke prevention in AF patients in China.

\section{Subjects And Methods}

\section{Patients and study design}


This was a retrospective study of prospectively obtained data from patients seen between January 2015 and December 2015 at a tertiary referral center in the East of China. Patients who diagnosed as Atrial fibrillation (AF) with an estimated glomerular filtration rate $\geq 30 \mathrm{ml} / \mathrm{min} / 1.73 \mathrm{~m}^{2}$ were recruited. The diagnosis of AF was based on the ESC guideline that at least 30 seconds electrocardiogram or $24 \mathrm{~h}$ Holter before or during hospitalization. Patients who uses warfarin irregularly or with INR value $<2$ were excluded. Finally, a total of 342 patients were recruited in this study. Then, according to the anticoagulation therapy, patients were divided into three groups including standard anti-coagulation (SAG) group, off-label (OFL) group and non-coagulation (NCG) group. Patients in SAG group received standard dosage of anti-coagulation therapy, including rivaroxaban $20 \mathrm{mg}$ once daily or dabigatran $110 \mathrm{mg} / 150 \mathrm{mg}$ twice daily, or warfarin with INR 2-3. Patients in OFL group received rivaoxaban < $20 \mathrm{mg}$ daily or dabigatran $110 \mathrm{mg} / 150 \mathrm{mg}$ once daily. Patients without anti-coagulation therapy were classified into NAG group. Patients were followed up every 6 months up to 5 years. All clinical events during followup including stroke and other thromboembolism events, acute coronary syndrome, major bleeding, allcause mortality and re-hospitalization were collected.

\section{Statistical analyses}

All analyses were carried out using SPSS 24.0. Descriptive statistics were used to evaluate patient related secondary objectives. For patient characteristics, two-sided Student's t-test, Chi square test, and Fisher's exact tests were utilized as appropriate. Comorbid conditions and responsible physician type were analyzed using Chi square test, and concomitant medication use was analyzed using Fisher's exact test. All statistical tests were two-sided and a p-value of less than or equal to 0.05 was considered statistically significant.

\section{Results}

\section{Baseline characteristics}

A total of 342 patients with AF were included into this study, 296 of which were regularly followed up while 46 were missed because of incorrect contact information. There were 148 patients in NAG group, 37 patients in OFL group and 111 patients in SAG group, respectively. The baseline characteristics of these three groups were summarized in Table 1. The $\mathrm{CHA}_{2} \mathrm{DS}_{2}$-VASc score of the NAG group, OFL group and SAG group were $3.6 \pm 1.8,3.7 \pm 1.7,3.5 \pm 1.7$, separately. Compared to OFL group $(71 \pm 10)$ and SAG group $(69 \pm 13)$, the age in NAG group was higher $(73 \pm 12)$. Unexpectedly, anti-platelet therapy was more common among patients in NAG group (49\% in NAG to $35 \%$ in OFL and $10 \%$ in SAG).

Figure 1 shows the anti-coagulation drugs used in OFL group (Fig. 1a) and SAG group (Fig. 1b). Most of the OFL group used rivaroxaban, while warfarin remained predominant in SAG group. 
Table 1

The Baseline characteristics of these 342 patients

\begin{tabular}{|lllll|}
\hline & NAG group & OFL group & SAG group & Pvalue \\
\hline Number of patients (n) & 148 & 37 & 111 & \\
\hline Male (\%) & 48 & 57 & 59 & 0.331 \\
\hline Age & $73 \pm 12$ & $71 \pm 10$ & $69 \pm 13$ & 0.036 \\
\hline Hypertension (\%) & 60 & 62 & 54 & 0.473 \\
\hline CHF (\%) & 17 & 22 & 30 & 0.074 \\
\hline DM (\%) & 31 & 38 & 20 & 0.114 \\
\hline Vascular disease (\%) & 31 & 30 & 20 & 0.114 \\
\hline Previous stroke/SE (\%) & 22 & 35 & 43 & 0.014 \\
\hline CHA ${ }_{2}$ DS ${ }_{2}$-VASc score & $3.6 \pm 1.8$ & $3.7 \pm 1.7$ & $3.5 \pm 1.7$ & 0.760 \\
\hline HAS-BLED score & $2.6 \pm 1.2$ & $2.3 \pm 1.3$ & $2.0 \pm 1.1$ & 0.001 \\
\hline Anti-platelet therapy (\%) & 49 & 35 & 10 & 0.000 \\
\hline SAG = standard anti-coagulation group, OFL = off-label group, NCG = non-anticoagulation group, CHF \\
\hline = congestive heart failure, DM = diabetes mellitus, SE = systemic embolism
\end{tabular}

\section{Events during follow-up}

We collected clinical events during our follow-up up to 5 years (Fig. 2). Compared to patients in SAG group, patients in OFL group had higher risk in stroke and thromboembolism events $(P=0.000)$. The risk of other events including major bleeding $(P=0.597)$ and re-hospitalization $(P=0.213)$ was similar in both groups, as well as the cardiovascular mortality $(P=0.640)$. Minor bleeding such as gingival bleeding and skin bleeding occurred more in SAG group. All adverse events were more common in NAG group except major bleeding.

As shown in Fig. 3, there was no significant difference on all-cause mortality between OFL and SAG (0.14 and $0.19, P=0.601)$, while these two groups were dramatically lower than NAG group $(0.52, P=0.000)$.

As shown in Fig. 4, the total cardiovascular events and mortality rate were not significant different between OFL group and SAG group $(P=0.863)$, while both were better than NCG group $(P=0.000)$.

\section{Subgroup analysis for anti-platelet therapy}

There were 73 patients using anti-platelet drugs such as aspirin and clopidogrel in NCG group, 75 patients without any anti-thrombotic therapy. We compared the anti-platelet subgroup with OFL group (Table 2). 
Off-label anti-coagulation therapy was not superior to prevent stroke or SE compared to anti-platelet therapy. The bleeding risk was higher in OFL group as well. Whereas, off-label anti-coagulation could decrease coronary events and all-cause mortality apparently.

Table 2

The compare between anti-platelet group and NCG

\begin{tabular}{|lllc|}
\hline & Anti-platelet subgroup & OFL group & P value \\
\hline Number of patients (n) & 74 & 35 & \\
\hline Stroke/SE (\%) & 15 & 17 & 0.707 \\
\hline Major bleeding (\%) & 0 & 6 & 0.039 \\
\hline ACS and HF (\%) & 22 & 0 & 0.007 \\
\hline Re-hospitalization (\%) & 78 & 69 & 0.270 \\
\hline Total cardiovascular mortality (\%) & 36 & 14 & 0.018 \\
\hline All-cause mortality (\%) & 47 & 14 & 0.001 \\
\hline OFL = off-label group, SE = systemic embolism, ACS = acute coronary syndrome, HF = heart failure \\
\hline
\end{tabular}

\section{Discussion}

AF causes severe economic burden and medical cost[10]. In east Asia, NOACs have been proved to reduce the burden of stroke and death compared to VKA[11]. Previous study have indicated that nonwhites as Asians with AF were at greater risk for anti-coagulation related intracranial hemorrhage (ICH)[5]. Therefore, some compromised dosage of NOACs are applied for the treatment of atrial fibrillation in east Asia, which is so called "Asian dose".

Evidence has showed the benefit of low dose rivaroxaban in east Asia[8, 12]. Low dose of NOACs have been proposed to decrease the risk of hemorrhage compared to standard dose of NOACs. However, Cheng. et a/pointed out that off-label rivaroxaban was associated with an increased risk of ischemic stroke with 2.75 times[6]. The standard dose NOACs was associated with a relatively mild stroke in the patients compared to under-dosed NOACs[13]. The previous studies were similar to our study which showed a poor clinical benefit of off-label anti-coagulation. The thrombosis risk was significantly higher in OFL group compared to SAG group. However, the thrombosis risk in OFL group was even higher than NAG group. This might be partly because of the survival period was shorter in NAG group. In addition, our results indicated that Asian dose of anti-coagulation therapy indeed did not lead to a less bleeding risk compared to the standard anti-coagulation therapy. In our study, standard dose of NOACs is only related to slightly increased risk of gingival bleeding and skin bleeding.

Here, our results show that the $\mathrm{CHA}_{2} \mathrm{DS}_{2}$-VASc score among the groups were close. The proportion of previous stroke and SE history were higher in OFL and SAG group while the age was higher in NAG group. 
This outcome is highly reasonable in clinic as the anti-thrombotic therapy are more active for stroke AF patients. The HAS-BLED score of NAG group was higher compared to other groups. That might be the reason why anti-coagulation therapy was not applied for these patients. For elder patients, anticoagulation needs more prudent due to the higher bleeding risk as well. In addition, rivaroxaban is used more commonly benefit from its various off-label dosage (10/15/20 mg per tablet).

Collectly, our study demonstrate that off-label anti-coagulation therapy can increase the survival rate of AF patients without decreasing the risk of stroke. We supposed that the major reason may be the reduction of coronary events, which are similar to a previous study in China[8]. Low dose rivaroxaban could reduce thrombus formation and platelet-dependent thrombin generation in patients with coronary heart disease, which are the potential ischemic benefits[14]. However, off-label NOACs brought higher bleeding risk without any benefits on thromboembolic events risk.

There were several limitations in our study. The sample size was relatively small, which may due to the loss of follow-up. In addition, the study was an observational study. More evidence such as randomized clinical trail is needed.

\section{Conclusion}

Asian dose of anti-coagulation therapy can not decrease the stroke risk of AF patients in east China. For the prevention of stroke and SE, standard dose of NOACs should be more appropriate for Asian AF patients. More experimental studies are needed to be carry out in the future.

\section{Declarations}

\section{Ethics approval and consent to participate}

All procedures performed in studies involving human participants were in accordance with the ethical standards of the institutional and/or national research committee and with the 1964 Helsinki declaration and its later amendments or comparable ethical standards. All patients have signed informed consent previously.

\section{Consent for publication}

Written informed consent for publication was obtained from all participants.

\section{Availability of data and materials}

The datasets used or analysed during the current study are available from the corresponding author on reasonable request.

\section{Competing interests}


The authors declare no conflicts of interest.

\section{Funding}

This study was supported by the National Natural Science Foundation of China (Grants 81800305).

\section{Authors' contributions}

J. Li and X.P. Luo designed research for this study; P. Zhou, R.C. Liu, Y.J. Yu, W. C, Y.Z. Ma, H.Y. Wang and M. Waresi collected samples and performed research; M. Wang analyzed data; P. Zhou and M. Wang wrote the manuscript.

\section{Acknowledgments}

The authors thank the information department of Huashan Hospital and Xunshu Company for giving help in collecting the data of the patients.

\section{References}

1. Wong, C.X., et al., Epidemiology of Atrial Fibrillation: The Australian and Asia-Pacific Perspective. Heart, Lung and Circulation, 2017. 26(9): p. 870-879.

2. Ceornodolea, A.D., R. Bal and J.L. Severens, Epidemiology and Management of Atrial Fibrillation and Stroke: Review of Data from Four European Countries. Stroke Res Treat, 2017. 2017: p. 8593207.

3. Hart, R.G., L.A. Pearce and M.I. Aguilar, Meta-analysis: antithrombotic therapy to prevent stroke in patients who have nonvalvular atrial fibrillation. Ann Intern Med, 2007. 146(12): p. 857-67.

4. Chan, Y.H., et al., Efficacy and Safety of Apixaban, Dabigatran, Rivaroxaban, and Warfarin in Asians With Nonvalvular Atrial Fibrillation. Journal of the American Heart Association, 2018. 7(8).

5. Shen, A.Y., et al., Racial/Ethnic Differences in the Risk of Intracranial Hemorrhage Among Patients With Atrial Fibrillation. Journal of the American College of Cardiology, 2007. 50(4): p. 309-315.

6. Cheng, W., et al., Low-Dose Rivaroxaban and Risks of Adverse Events in Patients With Atrial Fibrillation. Stroke, 2019. 50(9): p. 2574-2577.

7. Meng, S.W., et al., Direct Comparison of Low-Dose Dabigatran and Rivaroxaban for Effectiveness and Safety in Patients with Non-Valvular Atrial Fibrillation. Acta Cardiol Sin, 2019. 35(1): p. 42-54.

8. Lin, Y., et al., Effectiveness and Safety of Standard- and Low-Dose Rivaroxaban in Asians With Atrial Fibrillation. Journal of the American College of Cardiology, 2018. 72(5): p. 477-485.

9. Hindricks, G., et al., 2020 ESC Guidelines for the diagnosis and management of atrial fibrillation developed in collaboration with the European Association of Cardio-Thoracic Surgery (EACTS). European Heart Journal, 2020.

10. Chao, T.F., et al., Lifetime Risks, Projected Numbers, and Adverse Outcomes in Asian Patients With Atrial Fibrillation: A Report From the Taiwan Nationwide AF Cohort Study. Chest, 2018. 153(2): p. 453-466. 
11. Bai, Y., et al., Modelling projections for the risks related with atrial fibrillation in East Asia: a focus on ischaemic stroke and death. Europace, 2018. 20(10): p. 1584-1590.

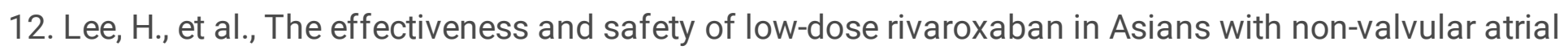
fibrillation. International Journal of Cardiology, 2018. 261: p. 78-83.

13. Jung, Y., et al., Stroke Severity in Patients on Non-Vitamin K Antagonist Oral Anticoagulants with a Standard or Insufficient Dose. Thrombosis and Haemostasis, 2018. 118(12): p. 2145-2151.

14. Geisler, T., et al., INHIBITORY MECHANISMS OF VERY LOW DOSE RIVAROXABAN IN NON-STELEVATION MYOCARDIAL INFARCTION. Journal of the American College of Cardiology, 2018. 71(11, Supplement): p. A1398.

\section{Figures}

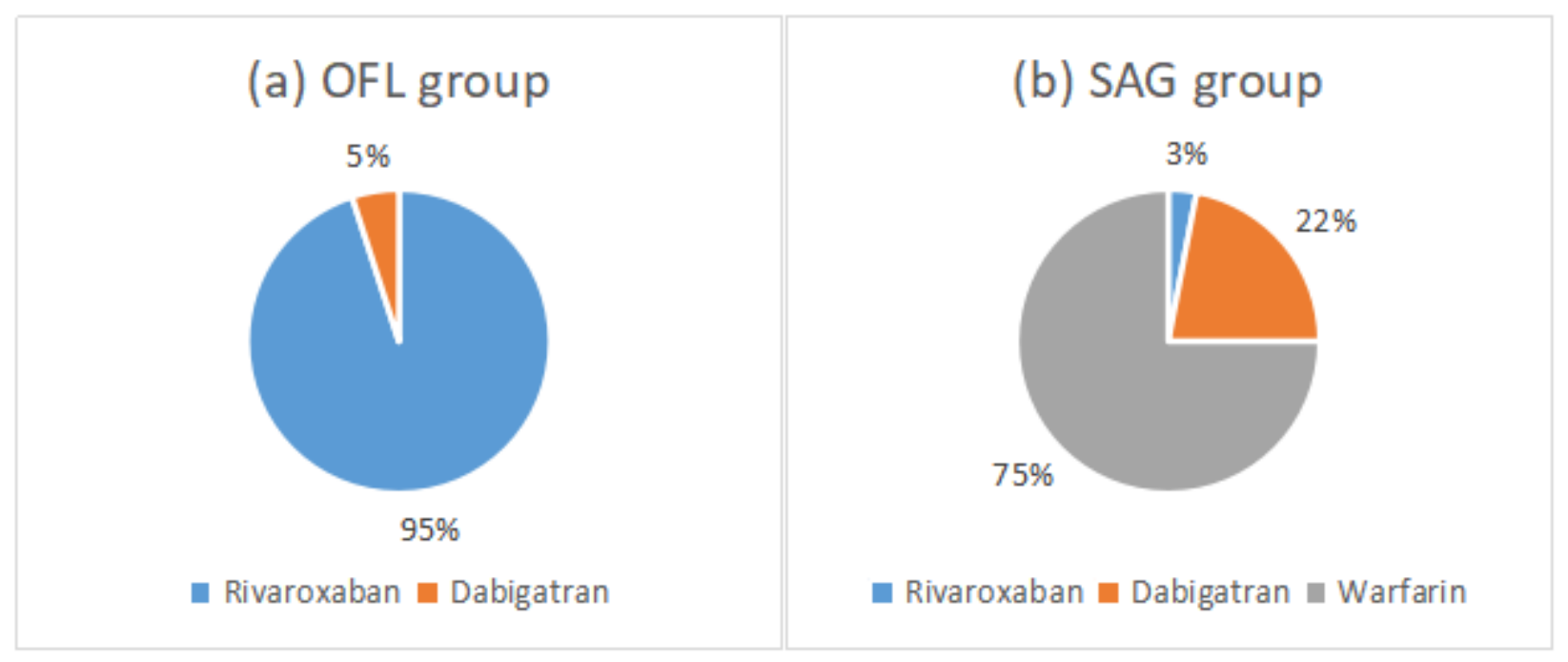

\section{Figure 1}

The anti-coagulation drugs used in OFL group (a) and SAG group (b) 


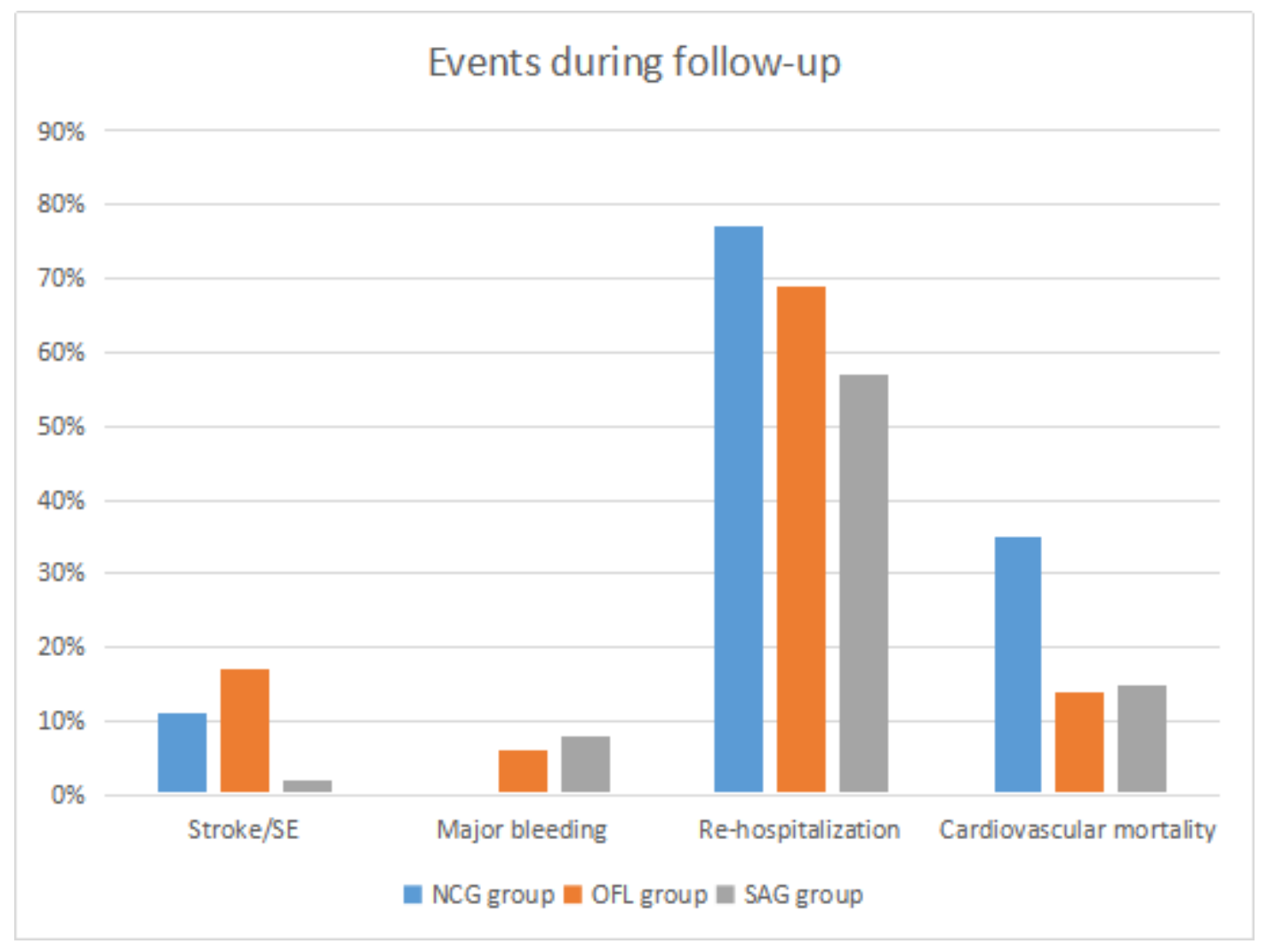

Figure 2

Clinical events during our follow-up up to 5 years 


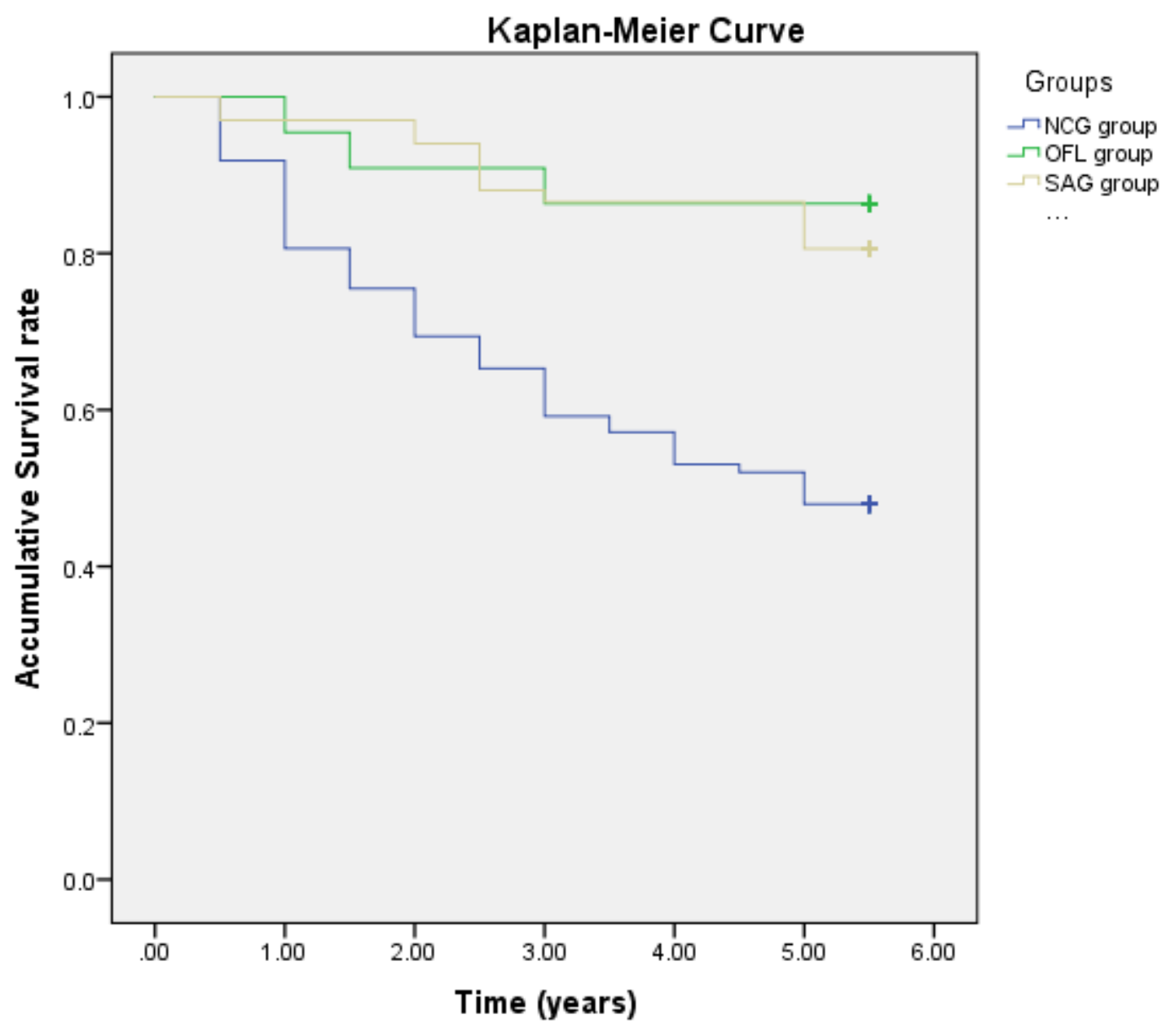

Figure 3

There was no significant difference on all-cause mortality between OFL and SAG (0.14 and 0.19, $P=0.601)$, while these two groups were dramatically lower than NAG group $(0.52, P=0.000)$. 


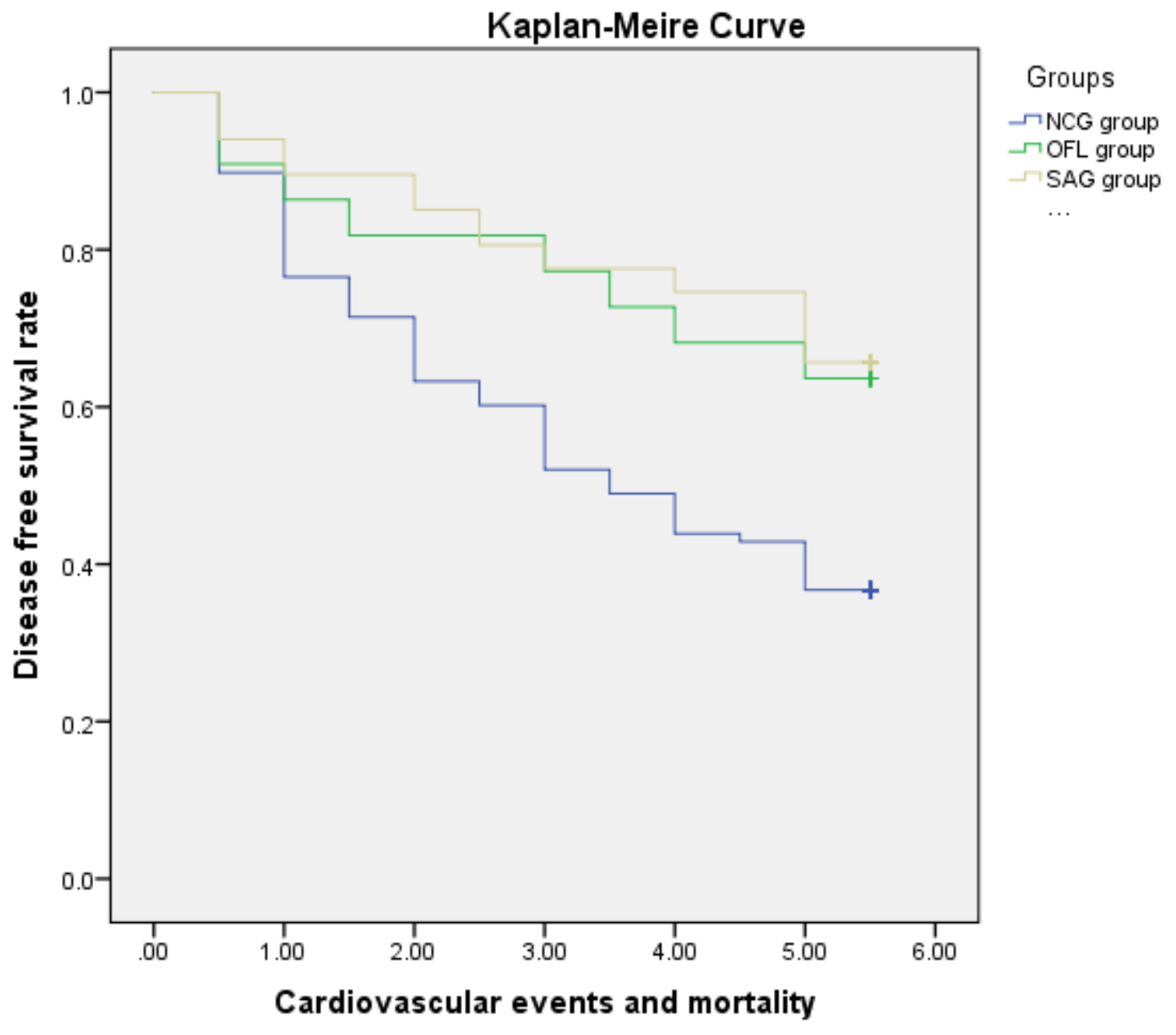

Figure 4

The total cardiovascular events and mortality rate were not significant different between OFL group and SAG group ( $P=0.863)$, while both were better than NCG group $(P=0.000)$. 